\title{
Correlation between Rigid Laryngoscopy and Histopathology of Laryngeal Lesions at Our Voice Clinic
}

\author{
${ }^{1}$ Nupur Kapoor Nerurkar, ${ }^{2}$ Sunil Garg \\ ${ }^{1}$ Consultant, Department of Laryngology, Bombay Hospital and Medical Research Center, Mumbai, Maharashtra, India \\ ${ }^{2}$ Senior Resident, Department of Laryngology, Bombay Hospital, Mumbai, Maharashtra, India
}

Correspondence: Nupur Kapoor Nerurkar, Consultant, Department of Laryngology, Bombay Hospital and Medical Research Center, Mumbai, Maharashtra, India, e-mail: nupurkapoor@yahoo.com

\section{ABSTRACT}

Introduction: In today's world of rapid technological advances, the medical field is developing at a rapid speed. Various sophisticated tools are available for the diagnostic work-up of patients with voice disorders. These are stroboscopy, optical coherence tomography (OCT), contact endoscopy and laryngeal USG, which gives sophisticated details of the larynx. However, at present most of these modalities are expensive and available only at few research centers. Rigid laryngoscopy is a noninvasive, easily available and fairly accurate diagnostic tool in patients with voice disorders.

Aims and objectives: The aim of our study was to assess the diagnostic potential of rigid laryngoscopy in different laryngeal lesions and its correlation with histopathology. This is a one year retrospective study at our voice clinic at Bombay Hospital, Mumbai.

Patients and methods: We examined 720 patients at our voice clinic from January 2008 to December 2008. Microlaryngeal surgery was performed on 59 of these. The clinical diagnosis was made after detailed history taking and clinical examination by a 70 degree Hopkins rod telescope.

Results: In our study, clinical diagnosis was $100 \%$ accurate in vocal fold subepithelial cyst, vocal fold nodules, laryngeal papilloma and contact granuloma. The clinical diagnosis had a reliability of $30 \%$ for Vocal fold polyps, $50 \%$ for leukoplakia, and $66 \%$ for malignant lesion. In suspected laryngeal polyps, leukoplakia and malignancy, our study indicates that we need to excise the lesion surgically and prove the histopathology.

Conclusion: Every tissue removed during laryngeal surgery should be sent for histopathology. Rigid Laryngoscopy is a safe and accurate tool to diagnose nodules and cysts.

Keywords: Hoarseness, Voice, Rigid laryngoscopy, Polyp, Cyst, Papilloma, Ulcer.

\section{INTRODUCTION}

In today's world of technological advances the medical field is developing at a rapid speed. Various sophisticated tools are available for the diagnostic work-up of patients with voice disorders. These are stroboscopy, optical coherence tomography (OCT), contact endoscopy, laryngeal USG, which gives sophisticated details of the larynx. However, at present most of these modalities are expensive and available only at few research centers. Rigid laryngoscopy is noninvasive, easily available and fairly accurate diagnostic tool in patients with voice disorders.

\section{AIMS AND OBJECTIVES}

Our aim in this study was to assess the diagnostic potential of rigid laryngoscopy in different laryngeal lesions and its corelation with histopathology. This is a one year retrospective study at our voice clinic at Bombay Hospital, Mumbai.

\section{MATERIALS AND METHODS}

We examined 720 patients at our voice clinic from January 2008 to December 2008. Microlaryngeal surgery was performed on 59 of these. The clinical diagnosis was made after detailed history taking and clinical examination by a 70 degree Hopkins rod telescope. This was a one year retrospective study at our voice clinic Bombay Hospital (tertiary care center), Mumbai. Tissue removed during laryngeal surgery was sent for histopathology.

\section{OBSERVATIONS (TABLES 1 AND 2)}

\begin{tabular}{lcll}
\hline Table 1: Clinically benign pathology (N=47) & $N$ \\
\hline $\begin{array}{lll}\text { Clinical } \\
\text { diagnosis }\end{array}$ & $N$ & HPE & \\
\hline Vocal fold polyp & 26 & $\begin{array}{l}\text { Vocal fold nodule } \\
\text { Vocal fold polyp } \\
\text { Squamous cell carcinoma }\end{array}$ & 12 \\
& & $\begin{array}{l}\text { Moderate dysplasia } \\
\text { Localized Reinke's edema }\end{array}$ & 01 \\
& & Ductal cyst & 05 \\
Vocal fold cyst & 05 & Vocal fold nodule & 06 \\
Vocal fold nodule & 06 & Squamous papilloma & 04 \\
Laryngeal papilloma & 04 & No dysplasia & 01 \\
Leukoplakia & 02 & Severe dysplasia & 01 \\
& & Mild focal dysplasia & 01 \\
Erythroplasia & 01 & Nodule & 01 \\
Contact lesion & 02 & Hyperkeratosis & 01 \\
& & Squamous mucosal & 01 \\
Ulcer & 01 & hyperplasia & \\
\hline
\end{tabular}


Table 2: Clinically malignant $(N=12)$

\begin{tabular}{cccccc}
\hline & Sq. cell ca. & Cyst & Nodule & Inflammatory polyp & Amyloidosis \\
\hline HPE & 8 & 1 & 1 & 1 & 1 \\
\hline
\end{tabular}

\section{RESULTS}

In our study, on rigid laryngoscopy, 47 patients (Fig. 1) were clinically diagnosed as having a benign lesions and 12 patients with malignant lesions. Out of 47 patients diagnosed with clinically benign lesions four turned out to be squamous cell carcinoma and two with carcinoma in situ on final histopathology.

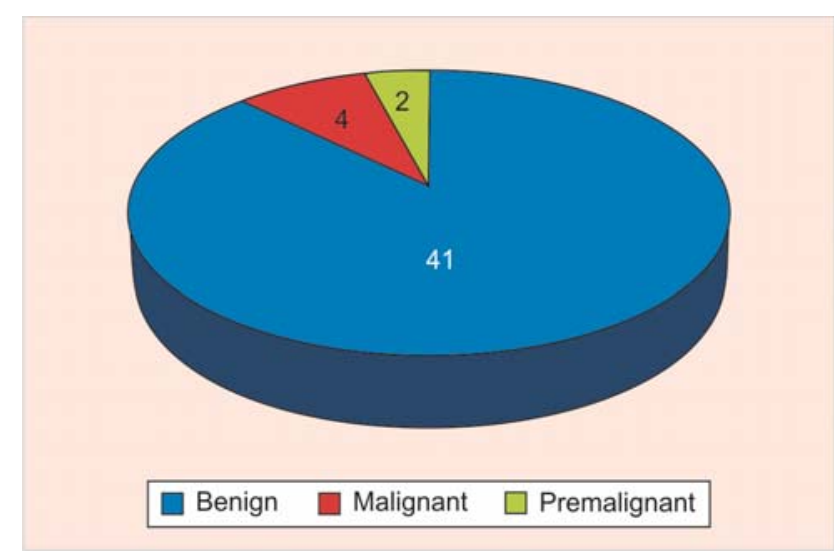

Fig. 1: Histopathology of clinically benign lesion $(\mathrm{N}=47)$

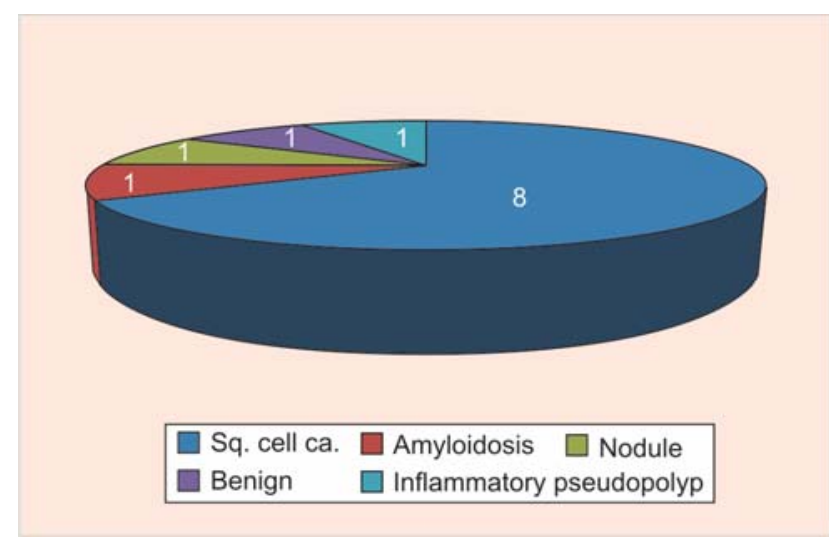

Fig. 2: Histopathology of clinically malignant lesion $(\mathrm{N}=12)$

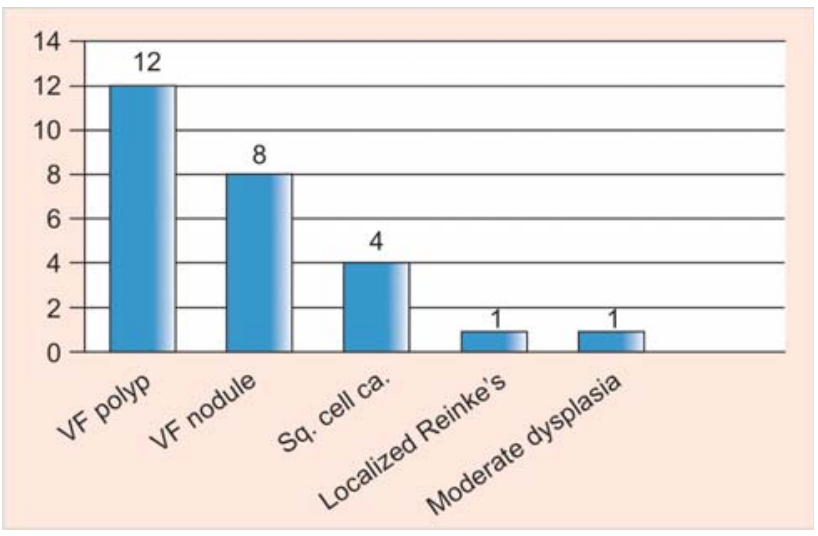

Fig. 3: Histopathology of clinically diagnosed VF polyp $(\mathrm{N}=26)$
In the 12 patients (Fig. 2) clinically diagnosed as having malignant lesions, four patients had a benign histopathology.

On rigid laryngoscopy, out of 47 patients clinically diagnosed as having benign lesions, 26 patients (Fig. 3) were diagnosed as vocal fold polyps. On histopathology, out of 26 patients, eight were polyps and the remaining were 12 vocal fold nodules, four squamous cell carcinoma, one moderate dysplasia and one localized Reinke's edema.

\section{INTERESTING CASES}

\section{Case 1 (Figs 4 and 5)}

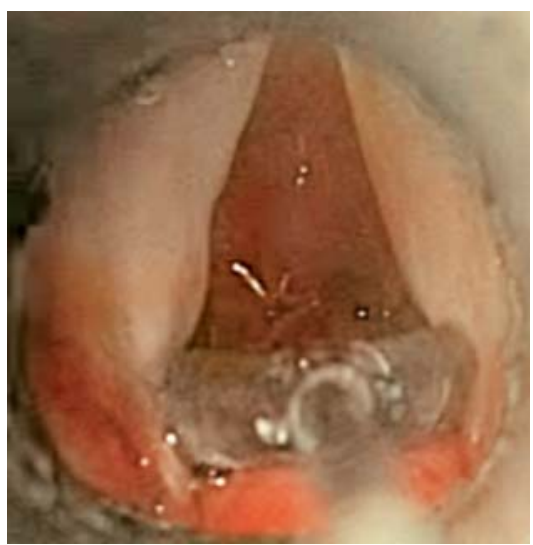

Fig. 4: Clinical diagnosis-malignant laryngeal lesion in a patient with history of previously treated adenocarcinoma of palate

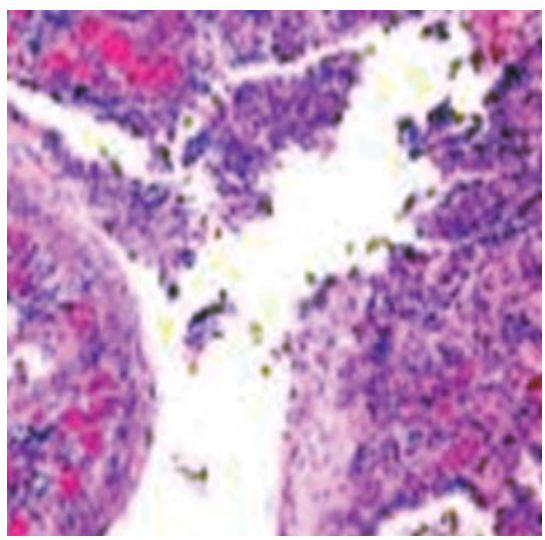

Fig. 5: HPE-inflammatory pseudopolyp probably as reaction to previous postoperative radiation therapy

\section{Case 2 (Figs 6 and 7)}

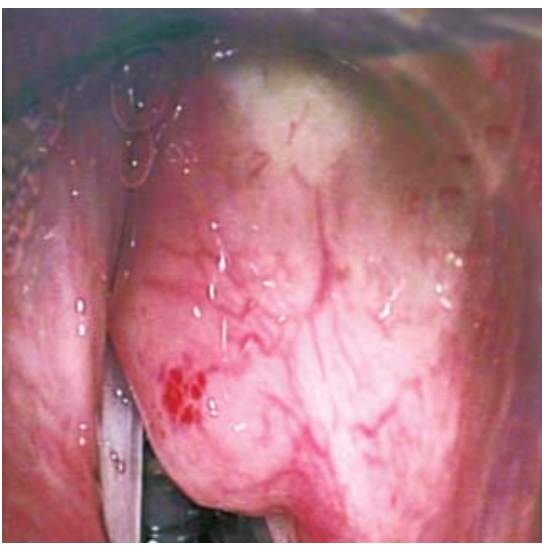

Fig. 6: Clinical diagnosis—right ventricular fold polyp 


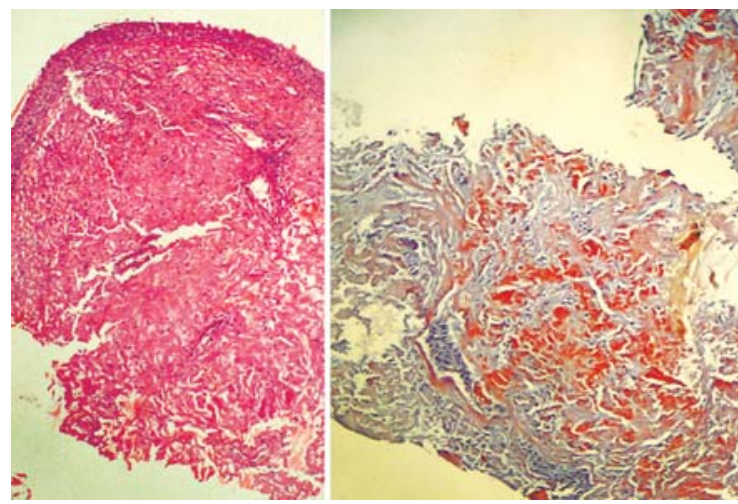

Fig. 7: HPE amyloidosis and showing congo red stain

\section{DISCUSSION}

The main concern of a patient with a voice disorder presenting to an ENT surgeon, is usually that of laryngeal cancer.

Laryngeal cancer cases are attributable to tobacco use, of which 75 and $12 \%$ are due to current and past smoking, respectively. Approximately 39\% are attributable to the interaction between alcohol and tobacco. ${ }^{1}$

Rigid laryngoscopy is an inexpensive tool to diagnose a patient with hoarseness of voice. If hoarseness of voice does not improve in 15 days the patient should visit an ENT surgeon.

Various sophisticated tools are available for the diagnostic work-up of patients with voice disorders. These are stroboscopy, videokymography, high speed photography, CT scan, MRI, optical coherence tomography (OCT), contact endoscopy and laryngeal USG, which give sophisticated details of the larynx.

By contact endoscopy (combination of autofluorescence and contact endoscopy) it is possible to observe the cells, nuclei, and cytoplasm, as well as different degree of abnormality (dysplasia grade I, II, III or CIN and laryngeal cancer). ${ }^{2}$

Optical coherence tomography (OCT) and high-frequency ultrasound are promising new methods in the early diagnosis of laryngeal cancer. Determination of epithelial thickness by OCT or high-frequency ultrasound may allow conclusions on whether or not a laryngeal lesion is malignant. ${ }^{3}$
However, at present most of these modalities are expensive and available only at few research centers. Rigid laryngoscopy is a noninvasive, easily available and fairly accurate diagnostic tool in patients with voice disorders.

In our study, clinical diagnosis was $100 \%$ accurate in vocal fold subepithelial cyst, vocal fold nodule, laryngeal papilloma and ulcer. The clinical diagnosis had reliability of 30\% for vocal fold polyp, 50\% for leukoplakia and 66\% for malignant lesion.

In laryngeal polyps, our study indicates that we had an accuracy of $30 \%$. In $70 \%$ of the cases the diagnosis was some other form of a benign lesion or a malignant tumor. Malignant laryngeal tumors when excised early (T1 and T2) need only regular follow-up and no further treatment, decreasing the morbidity of the patient dramatically. These laryngeal lesions when detected early are managed endoscopically with preservation of voice. Every tissue removed during laryngeal surgery should be sent for histopathology.

\section{CONCLUSION}

From our study, we conclude that rigid laryngoscopy examination alone gives $100 \%$ accuracy in case of clinically suspected nodules, cysts and papillomas. Therefore, it is justified to follow a conservative approach in these situations if the lesion is one that will respond to speech therapy, such as vocal fold nodules or if surgery is associated with a high risk.

We can be conservative with vocal fold nodules and cysts. We should be aggressive in the management of vocal fold polyps.

\section{REFERENCES}

1. Hashibe M, Zaridze D, et al. Contribution of tobacco and alcohol to the high rates of squamous cell carcinoma of the supraglottis and glottis in Central Europe. Am J Epidemiol 1 Apr 2007;165(7):814-20. Epub 2007 Jan 22.

2. Ahrens C, Glanz H, et al. Contact endoscopy of larynx. Ann Otol Rhinol Laryngol Feb 2003;112(2):113-19.

3. Kraft M, Luerssen $\mathrm{K}$, et al. Mucosal lesions in the larynx: Predictive value of new imaging modalities for a histological diagnosis. www.pubMed.com (PIMD: 17928978). 